\title{
Reduction of High Cholesterol Levels by a Preferably Fixed-Combination Strategy as the First Step in the Treatment of Hypertensive Patients with Hypercholesterolemia and High/Very High Cardiovascular Risk: A Consensus Document by the Italian Society of Hypertension
}

\author{
Guido Grassi ${ }^{1}$ - Rita Del Pinto ${ }^{2}$. Claudia Agabiti Rosei ${ }^{3}$. Davide Agnoletti ${ }^{4}$. Claudio Borghi ${ }^{4}$. Arrigo F. G. Cicero ${ }^{4}$. \\ Carolina De Ciuceis ${ }^{3}$. Giovambattista Desideri ${ }^{2}$. Davide Grassi ${ }^{2}$ - Maria Lorenza Muiesan ${ }^{3}$. Anna Paini ${ }^{3}$.

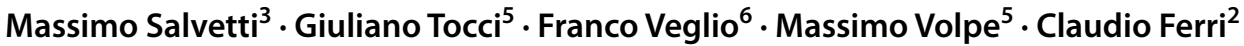

Received: 10 November 2021 / Accepted: 22 December 2021 / Published online: 3 January 2022

(c) The Author(s) 2021

\begin{abstract}
The primary and secondary prevention strategies of atherosclerotic cardiovascular disease (ASCVD) largely rely on the management of arterial hypertension and hypercholesterolemia, two major risk factors possibly linked in pathophysiological terms by the renin-angiotensin system activation and that often coexist in the same patient synergistically increasing cardiovascular risk. The classic pharmacologic armamentarium to reduce hypercholesterolemia has been based in the last two decades on statins, ezetimibe, and bile acid sequestrants. More recently numerous novel, additive resources targeting different pathways in LDL cholesterol metabolism have emerged. They include drugs targeting the proprotein convertase subtilisin/kexin type 9 (PCSK9) (inhibitory antibodies; small-interfering RNAs), the angiopoietin-like protein 3 (inhibitory antibodies), and the ATP-citrate lyase (the inhibitory oral prodrug, bempedoic acid), with PCSK9 inhibitors and bempedoic acid already approved for clinical use. With the potential of at least halving LDL cholesterol levels faster and more effectively with the addition of ezetimibe than with high-intensity statin alone, and even more with the addition of the novel available drugs, this document endorsed by the Italian Society of Hypertension proposes a novel paradigm for the treatment of the hypertensive patient with hypercholesterolemia at high and very high ASCVD risk. Our proposal is based on the use as a first-line of a preferably fixed combination of lipid-lowering drugs, under the motto "Our goal: achieve control. No setback: combine and check".
\end{abstract}

Keywords Hypertension · Hypercholesterolemia · Fixed combination · Cardiovascular prevention

Guido Grassi and Rita del Pinto: equal contribution.

Claudio Ferri

claudio.ferri@univaq.it

Clinica Medica, University of Milano Bicocca, Milan, Italy

2 Department of Clinical Medicine, Public Health, Life and Environmental Sciences; Internal Medicine and Nephrology Unit - San Salvatore Hospital, University of L'Aquila, 67100 L'Aquila, Italy

3 Internal Medicine, Department of Clinical and Experimental Sciences, University of Brescia, Brescia, Italy
4 Department of Medical and Surgical Sciences, University of Bologna, Bologna, Italy

5 Department of Clinical and Molecular Medicine, University of Rome Sapienza, Rome, Italy

6 Department of Medical Sciences, University of Turin, Turin, Italy 


\section{Introduction}

In the modern approach to the management of cardiovascular diseases, an effective control of risk factors is of paramount importance. Over the past 20 years, several effective therapeutic strategies have been developed for the management of cardiovascular risk factors to prevent the onset and progression of atherosclerotic cardiovascular disease (ASCVD). However, ASCVD remains a leading cause of death worldwide [1]. Arterial hypertension and hypercholesterolemia are the main risk factors for cardiovascular diseases, and their management is a mainstay of primary and secondary ASCVD prevention [2]. Population epidemiology demonstrates that these two risk factors often cluster in the same patient and synergistically increase the risk of cardiovascular diseases: in fact, the reported rates of ASCVD events are higher when these risk factors coexist compared to what would be expected considering the impact of the two risk factors separately [3]. Arterial hypertension and hypercholesterolemia are also strongly correlated in pathophysiological terms. The mechanisms responsible for their interaction have not yet been fully elucidated, but there is evidence of an involvement of the renin-angiotensin-aldosterone system (RAAS) [4]. In particular, hypercholesterolemia acts on the genetic control of the type 1 angiotensin II receptor $\left(\mathrm{AT}_{1}\right)$ by increasing the stability of its messenger RNA, with consequent structural hyper-expression of vascular $\mathrm{AT}_{1}$ receptors that determines an exaggerated response to angiotensin II in hypercholesterolemic patients [4]. This phenomenon, in turn, promotes or exacerbates hypertension and the development of ASCVD. In therapeutic terms, while the treatment of hypercholesterolemia and hypertension is mandatory to improve the individual risk profile, especially by using combination strategies with proven benefit (e.g. statins and RAAS blockers) [5], a novel paradigm is now emerging grounded on the evidence-based assumption that low-density lipoprotein (LDL) cholesterol reduction follows the principle "the lower the better" for maximal benefits in cardiovascular protection. In fact, the direct and positive correlation of ASCVD risk reduction with incrementally lower LDL cholesterol values suggests that an intensive management of hypercholesterolemia by means of a first-line combination therapy could represent a particularly successful strategy in individuals with concomitant hypertension. This document, endorsed by the Italian Society of Hypertension, summarizes the state of the art for the treatment of hypercholesterolemia and proposes a novel approach to the management of the hypertensive and hypercholesterolemic patients aimed at reducing the disease burden and its devastating socioeconomic implications.

\section{Traditional and Novel Lipid Lowering Drugs}

The classic armamentarium against hypercholesterolemia, which only consisted for a long time in hydroxymethylglutaryl-coenzyme A (HMG-CoA) reductase inhibitors, more simply known as statins, but also in ezetimibe and bile acid sequestering resins, has recently been enriched with numerous resources, some of which have already been recommended in the management of hypercholesterolemic patients, such as the inhibitors of proprotein convertase subtilisin/kexin type 9 (PCSK9) [6], and other ones at an advanced stage of evaluation or already approved by regulatory authorities, such as inclisiran, evinacumab and bempedoic acid [7-9].

\subsection{Statins}

Statins reduce hepatic synthesis of cholesterol by competitively inhibiting the enzyme HMG-CoA reductase, the ratelimiting enzyme of cholesterol biosynthesis. The reduction of intracellular cholesterol promotes greater expression of the LDL receptor (LDLR) on the surface of hepatocytes, resulting in an increase in the uptake of circulating LDL cholesterol, with a decrease in the relative plasma concentration $[10,11]$. Since the biosynthesis of cholesterol follows a circadian trend, with an increase in the night hours [12], the differences in the half-life of the various statins account for the opportunity of evening administration for molecules with a shorter half-life, such as simvastatin [13]. Conversely, statins with a longer half-life, such as atorvastatin, can also be taken in the morning without loss of cholesterol-lowering efficacy [14]. The extent of the reduction in LDL cholesterol depends on the type of statin and its dosage [10,11]. A high-intensity regimen is defined as the statin dose that, on average, reduces LDL cholesterol by at least $50 \%$; a moderate intensity regimen can reduce it by $30-50 \%$, while a low intensity regimen reduces LDL cholesterol up to $30 \%$ [10, $11]$.

In terms of efficacy, there is considerable interindividual variation in LDL cholesterol reduction with the same statin dose, and this appears to be mostly related to genetic background [15]. This feature and, to an even greater extent, poor adherence and persistence in therapy are the main causes of the poor response to statin treatment $[16,17]$. The main reason for the discontinuation of statin therapy is intolerance, which largely coincides with the appearance of muscle symptoms (statin-associated muscle symptoms, SAMS) [18]. According to observational and clinical studies, statin intolerance would affect a variable percentage of patients, ranging from 10 to $30 \%$ [16]. From a mechanistic point of view, the muscular toxicity of 
statins is believed to be the consequence of the non-selective and tissue-specific inhibition of HMG-CoA reductase, an effect to which skeletal muscle is 40 times more sensitive than hepatocytes and myocardium [18]. There is evidence of common genetic variants strongly associated with an increased risk of statin-induced myopathy. This is the case of the single nucleotide polymorphism (SNP) in the SLCO1B1 gene encoding the organic anion transporter polypeptide OATP1B1, involved in the regulation of hepatic uptake of statins [19], as well as a variant of the LILRB5 gene (leukocyte immunoglobulin-like receptor subfamily-B) associated with inhibition of immunemediated repair and regeneration of skeletal muscles and with elevated levels of lactate dehydrogenase (LDH) and creatine phosphokinase (CPK) [20]. It is worth noting, however, that in several large randomized controlled trials, the excess risk of myopathy relative to placebo was extremely low with all marketed statins at up to maximum recommended doses. On the other hand, even the incidence of a generic intolerance was similar between active treatment and placebo [11].

As a consequence, adherence to and persistence on statin therapy remain largely perfectible. Estimates suggest that $40-75 \%$ of patients discontinue statin therapy within one year of initiation, with higher rates of discontinuation in primary than secondary prevention in elderly patients (> 75 years), in women, and among those taking complex therapeutic regimens due to comorbidities [21]. In Italy, statins belong to the therapeutic categories with the highest percentages $(41.6 \%)$ of reduced adherence (therapeutic coverage $<40 \%$ ), especially among individuals aged between 65 and 84 years (42.3\%) [22]. Similarly, data on persistence to treatment indicate a $50 \%$ probability of discontinuing treatment as early as 150 days from the start of therapy (with a mean of 130 days for women and 180 days for men), with a maximum peak in the age group between 55 and 64 years old. About a fifth of users stop the therapy after one month from the start and only a third of new users are still on treatment 1 year after the start of therapy (men: 38\%; women: 29\%) [22].

In consideration of the unfavorable impact of the interruption of cholesterol-lowering therapy in the patient at high cardiovascular risk, the currently recommended approach to the patient with statin intolerance remains centered around the active involvement of the patient, for a shared decision-making process in case of restoration of the therapy. Pharmacological options include discontinuation and subsequent resumption of treatment with the same or another statin, substitution with another type of statin, gradual dose reduction and administration every other day, and the use of other lipid-lowering strategies in case failure (ezetimibe in the first instance, then the bile acid sequestrants) $[18,23]$.

\subsection{Ezetimibe}

By targeting the cholesterol transport protein Nieman Pick C1-like 1 protein (NPC1L1P) at the jejunal enterocyte brush border, ezetimibe inhibits LDL cholesterol absorption [24], thereby reducing the amount of cholesterol delivered to the liver. As a consequence, LDLR expression in the liver is upregulated, leading to increased clearance of LDL cholesterol from the bloodstream. The recommended dose of ezetimibe $(10 \mathrm{mg} / \mathrm{day})$ can be administered in the morning or evening irrespective of food intake, with no required adjustment in the presence of mild liver impairment or mildto-severe renal insufficiency [11].

Clinical studies in hypercholesterolemic individuals indicate that monotherapy with ezetimibe reduces LDL cholesterol by $15-22 \%$ [11]. When added to statins, ezetimibe was found to reduce LDL cholesterol levels up to an additional $21-27 \%$ [25]. The additive benefit was also reported for its use in combination with bile acid sequestrants and PCSK9 inhibitors (PCSK9i) [26, 27]. In parallel, the addition of ezetimibe to simvastatin in stable patients with recent acute coronary syndrome reduced the composite outcome of cardiovascular death, major coronary events, or nonfatal stroke by an additional $2 \%$ in the long term [28]. Specifically, in the Improved Reduction of Outcomes: Vytorin Efficacy International Trial (IMPROVE-IT), fewer events (32.7 vs. $34.7 \%, p=0.016)$ were recorded in the group of individuals taking simvastatin $(40 \mathrm{mg})$ plus ezetimibe $(10 \mathrm{mg})(\mathrm{N}$. 9067) as compared to the group of individuals receiving the statin monotherapy (N. 9077). The benefit was observed also among patients already treated with statins. The average LDL cholesterol levels during the study were $55 \mathrm{mg} / \mathrm{dL}$ in patients taking the combination of ezetimibe and simvastatin, and $70 \mathrm{mg} / \mathrm{dL}$ in the simvastatin group [28].

According with the observed safety and efficacy of ezetimibe from clinical trial data, genetic studies of mutations in NPC1L1 indicate that naturally occurring mutations that inactivate the protein are associated with reduced LDL cholesterol levels and lower risk for coronary artery disease [29].

\subsection{PCSK9-Inhibitors}

In recent years, the first two drugs with a new mechanism of action for the treatment of hypercholesterolemia, evolocumab and alirocumab, have become available. These are monoclonal antibodies with an inhibitory action against the serine protease PCSK9, involved in the recycling of the membrane receptor for LDL lipoproteins [6]. By binding the enzyme with an approximate ratio of $1: 1$, they prevent its interaction with the LDL cholesterol-receptor complex and the lysosomal degradation of the LDL receptor [30]. Consequently, LDL receptors can recirculate on the surface 
of hepatocytes up to approximately 150 times, increasing the clearance of LDL cholesterol [31]. Given the mechanism of action, these drugs are effective in reducing LDL cholesterol in all patients who express the relative receptor in the liver, with variability of response in patients with homozygous familial hypercholesterolemia in relation to residual receptor expression [32]. The net clinical effect is the average reduction of $60 \%$ in serum LDL cholesterol levels when used alone, which rises up to $85 \%$ when combined with highintensity statin and ezetimibe [11], with a benefit on the risk of cardiovascular events by a magnitude of $15-20 \%$ [33, 34]. In Italy, the primary reason (53.2\%) for prescribing a PCSK9i is intolerance to statins, and not failure to achieve the LDL cholesterol targets set for the specific risk profile [22].

\subsection{PCSK9 Small-Interfering RNA}

Inclisiran is a new drug authorized in 2020 in Europe for the treatment of adults with heterozygous familial and unfamiliar hypercholesterolemia or mixed dyslipidemia, who are not controlled by statin at the maximum tolerated dose or are intolerant to statin. It is a small-interfering RNA (siRNA), administered subcutaneously with an initial dose, repeated at 3 months and then every 6 months, which reduces the hepatic synthesis of PCSK9 by selectively and specifically degrading its messenger RNA (mRNA), with a prolonged effect up to 84 days after administration $[8,35]$. A single catalytic complex is capable of degrading numerous transcripts, which is important during statin therapy, known to induce increased PCSK9 production [8]. In ORION-1, inclisiran effectively and sustainably lowered LDL cholesterol $(-52.6 \%$ at 6 months at $300 \mathrm{mg}$ twice yearly doses compared with placebo, $p<0.001)$ in adults $(>18$ years) with baseline LDL cholesterol values $>70 \mathrm{mg} / \mathrm{dL}$ in the presence of ASCVD or $>100 \mathrm{mg} / \mathrm{dL}$ in its absence, who were already on maximal statin therapy with or without further cholesterol-lowering therapy, excluding PCSK9 inhibitors [36, 37]. A similar and sustained reduction in LDL cholesterol was confirmed in the phase III studies ORION-10 and ORION11 ( $-52.3 \%$ and $-49.9 \%$ at 510 days, respectively), conducted in over 3000 hypercholesterolemic patients with ASCVD or equivalent [37]. Low interindividual variability in the effect of inclisiran during treatment was described: at 510 days, over $85 \%$ and $65 \%$ of treated patients had a reduction in LDL cholesterol of at least $30 \%$ and $50 \%$, respectively [37]. The most common adverse effect $(\leq 5 \%$ of participants), however minor, was represented by local reactions at the injection site [37]. Additional common adverse events (> $2 \%$ of patients) included myalgia, headache, fatigue, nasopharyngitis, low back pain, hypertension, diarrhea and dizziness [36]. The impact of inclisiran on cardiovascular events is being evaluated in the ORION-4 study [38].

\subsection{ANGPTL3 Inhibition}

Evinacumab is a fully human monoclonal antibody with inhibitory effect on angiopoietin-like protein 3 (ANGPTL3) $[39,40]$, the gene of which is predominantly expressed in the liver [41]. ANGPTL3 is an inhibitor of lipoprotein and endothelial lipase with a key role in the metabolism of lipids, of which it increases the circulating levels [41]. Loss-offunction gene variants have been associated with low LDL cholesterol and triglyceride levels, as well as a $41 \%$ lower risk of coronary heart disease than the wild type gene [42]. Both the genetic and the therapeutic effect are independent of the LDL receptor [43].

In a phase 2 study of nine patients with homozygous familial hypercholesterolaemia, treatment with evinacumab resulted in an average 49\% reduction in LDL cholesterol levels after 4 weeks from baseline [44]. This effect was confirmed in the phase 3 Evinacumab Lipid Studies in Patients with Homozygous Familial Hypercholesterolemia (ELIPSE $\mathrm{HoFH}$ ), conducted in 65 patients with homozygous familial hypercholesterolemia already on stable lipid-lowering therapy, randomized in a 2:1 ratio to receive an intravenous infusion of evinacumab ( $15 \mathrm{mg} / \mathrm{kg}$ body weight) every 4 weeks or placebo [39]. At week 24, LDL cholesterol was reduced by $47.1 \%$ from baseline in patients treated with evinacumab, while it was increased by $1.9 \%$ in the placebo group (mean percentage and absolute difference between groups: $-49.0 \%, p<0.001$ and $-132.1 \mathrm{mg} / \mathrm{dL}, p<0.001$, respectively) [39]. Evinacumab was overall well tolerated: no one discontinued the drug due to side effects, and the most common adverse event was represented by a flu-like illness (11\% of patients); a slight and transient increase in transaminases occurred in 5\% of patients treated with evinacumab and in $10 \%$ of those assigned to placebo. Patients treated with evinacumab did not develop anti-drug antibodies [39].

\subsection{Bempedoic Acid}

Bempedoic acid is the first of a new class of non-statin lipidlowering drugs that inhibits hepatic cholesterol biosynthesis at the level of ATP-citrate lyase, two steps upstream of the statin target (HMGCoA reductase), reducing LDL cholesterol levels and at the same time increasing the expression of the relative receptor [7]. Administered as a prodrug orally once daily, it is converted into active form by enzymes that are located specifically in the liver and are not found in the muscle [7]. The lack of active metabolites in the skeletal muscle makes bempedoic acid a promising alternative for patients with SAMS [7].

The efficacy and safety of bempedoic acid-either as a monotherapy versus placebo or other lipid-lowering drugs, or in combination with ezetimibe-have been 
examined in different clinical scenarios as part of the CLEAR (Cholesterol Lowering via Bempedoic Acid, an ACL-inhibiting Regimen) clinical research program [45], which showed that bempedoic acid is able to reduce LDL cholesterol levels up to almost $30 \%$ in monotherapy and over $40 \%$ in combination with ezetimibe. Awaited are the results of the CLEAR Outcomes study (Evaluation of Major Cardiovascular Events in Patients With, or at High Risk for, Cardiovascular Disease Who Are Statin Intolerant Treated With Bempedoic Acid (ETC-1002) or Placebo), aimed at evaluating the effect of bempedoic acid on cardiovascular morbidity and mortality [46].

In the CLEAR Tranquility study (bempedoic acid 180 $\mathrm{mg} /$ day vs placebo in statin intolerant patients with LDL cholesterol $\geq 100 \mathrm{mg} / \mathrm{dL}$ on ezetimibe $10 \mathrm{mg} /$ day), bempedoic acid reduced LDL cholesterol by $28.5 \%$ compared with placebo at 12 weeks, with also a favorable effect on the non-LDL cholesterol profile $(-23.6 \%)$ and on the $\mathrm{C}$ reactive protein $(\mathrm{CRP} ;-31.0 \%)(p<0.001$ for all comparisons) [47]. Similarly, in the CLEAR Serenity study (bempedoic acid $180 \mathrm{mg} /$ day with or without additional lipid-lowering therapy in patients intolerant to at least 2 statins), the new drug resulted in a $21.4 \%$ reduction in LDL cholesterol compared to placebo at 12 weeks, significantly improving also the non-LDL lipid profile and serum CRP levels [48]. The CLEAR Wisdom and CLEAR Harmony studies then confirmed the cholesterol-lowering effect of bempedoic acid in patients at high cardiovascular risk with manifest atherosclerotic disease, heterozygous familial hypercholesterolemia or both conditions $[49,50]$. The aggregate analysis of the same studies indicated a good tolerability and safety profile for bempedoic acid [45]. Adverse events leading to treatment discontinuation occurred at rates of 13.4/100 person-years for bempedoic acid and 8.9/100 person-years for placebo, with the most common cause being myalgia, whose frequency, however, was not dissimilar between drug and placebo [45]. The use of bempedoic acid was also associated with mild and reversible laboratory alterations (increase in urea nitrogen, creatinine and uric acid; decrease in hemoglobin levels) and a higher incidence of gout compared with placebo (1.6/100 versus $0.5 / 100$ person-years, respectively) [45]. Conversely, there were fewer cases of incident diabetes with bempedoic acid than with placebo $(4.7 / 100$ versus 6.4/100 person-years).

The drug has been approved, both as a monotherapy and in combination with ezetimibe, for use in adults with heterozygous familial hypercholesterolaemia or overt ASCVD requiring further reduction of LDL cholesterol in addition to diet and statin therapy at the maximally tolerated dose, or with documented statin intolerance.

\section{Proposed Approach to the Hypertensive and Hypercholesterolemic Patient}

The scenario depicted above clearly indicates that several novel weapons are available in the fight against hypercholesterolemia, which overcome some major limitations of the traditional drugs and extend their efficacy. In addition, the role of lifestyle modification has been extensively confirmed as adjuvant in the management of cardiovascular risk $[51,52]$. These treatment opportunities must be fully deployed in order to act incisively on this major cardiovascular risk factor-and hypertension bad companionwhich carries devastating socio-economic consequences.

In agreement with this, current guidelines recommend the prompt and active management of risk factors in individuals who are at high or very high total ASCVD risk for established disease, diabetes, moderate-severe chronic kidney disease, familial hypercholesterolemia, grade 3 hypertension, or severe hypercholesterolemia (total cholesterol $>310 \mathrm{mg} / \mathrm{dL}$, LDL cholesterol $>190 \mathrm{mg} / \mathrm{dL}$ ), as well as in cases when the combination of several risk factors results in high levels of estimated ASCVD risk using validated risk assessment systems, such as the calibrated country-specific versions of the SCORE (Systematic Coronary Risk Estimation) system [11, 53]. Guidelines also emphasize that the presence of hypertension-mediated organ damage (HMOD), including, but not limited to, hypertensive left ventricular hypertrophy, microalbuminuria, elevated albumin-creatinine ratio, advanced retinopathy, and arterial stiffening (e.g. pulse pressure $\geq 60$ $\mathrm{mmHg}$ in the elderly), increases the total ASCVD risk beyond the SCORE estimates, even more so if multiple and/or pronounced HMODs are present [54]. Without taking into account this important aspect, in fact, the patient with hypertension is exposed to misclassification in lower risk categories and to consequent therapeutic inertia, which might have detrimental long-term consequences, especially in the presence of other hidden and/or major ASCVD risk factors, such as elevated hyperuricemia and/ or high LDL cholesterol levels, respectively [1-3, 55].

Thus, we propose that, in the patient with hypertension and concomitant hypercholesterolemia who is classified as having high or very high ASCVD risk after a complete risk stratification that also accounts for HMOD, lifestyle intervention is warranted and the lipid-lowering combination therapy based on high intensity statin, at the highest tolerated dose, plus ezetimibe is offered in the first instancepreferably as a fixed combination-with the aim of rapidly achieving the recommended LDL cholesterol target and the reduction in LDL cholesterol levels of at least $50 \%$ [11] (Fig. 1). In fact, the average LDL cholesterol reduction achieved with high intensity statin (about 50\%) rises 


\section{A. Former algorithm}

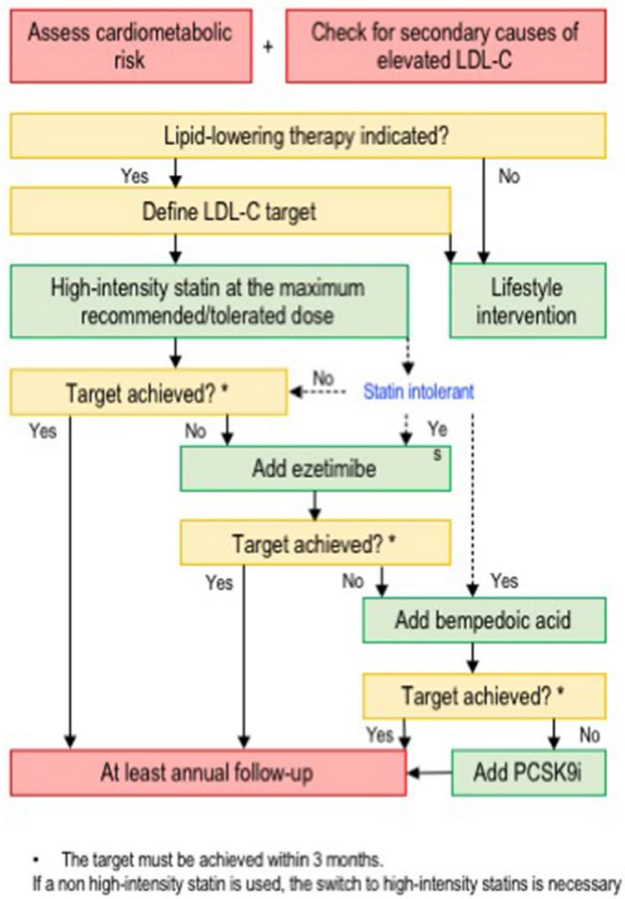

If a non high-intensity statin is used, the switch to $h$

\begin{abstract}
(a)
\end{abstract}
Fig. 1 Current strategy (A) and novel proposed approach (B). The use of a (preferably fixed) combination containing two drugs, i.e. a high intensity statin at the highest tolerated dose + ezetimibe, is proposed as the first step in the management of LDL-hypercholesterolemia in the high- and very high-risk hypertensive individual. Bempedoic acid is proposed in all statin intolerant patients and in those who did not reach the LDL cholesterol target with lifestyle changes + high inten-

to about $65 \%$ with the addition of ezetimibe [11]. If this goal is not attained, or if statin intolerance is documented, then anti-PCSK9 drugs and/or bempedoic acid should be promptly added to the combination of high intensity statin plus ezetimibe or be considered together with ezetimibe in the first instance, respectively (Fig. 1). It is estimated that PCSK9 inhibitors improve the lipid-lowering efficacy of high intensity statin plus ezetimibe by an additional $20 \%$, for a total effect of about $85 \%$, which is particularly relevant when a target LDL cholesterol of at least $55 \mathrm{mg}$ / $\mathrm{dL}$ is required, such as in the very high-risk patient [11].

The proposed approach could be advantageous both in the primary and in the secondary prevention of ASCVD in the high- and very high-risk hypertensive and hypercholesterolemic patients. In fact, it would allow the rapid control of LDL cholesterol and disrupt the culprit molecular events involved in its intertwined relationship with hypertension, thereby reducing the risk of disease development, the progression of subclinical damage, and the recurrence of major events. Obviously, a similar approach—based on the use of fixed combinations containing either a high intensity statin

\section{B. Novel algorithm}

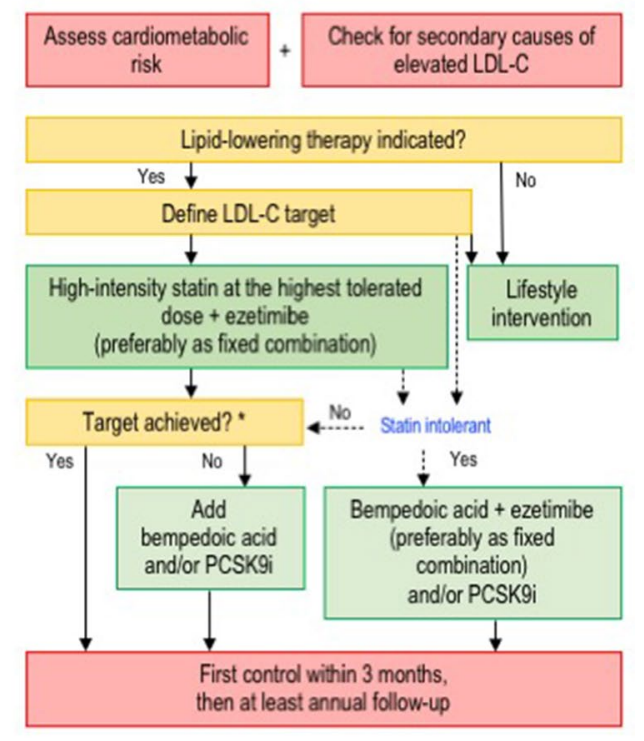

sity statin + ezetimibe. The addition of bempedoic acid to some of the non-high intensity statins can provoke interferences and must be avoided. The same approach may be considered in low to moderate risk hypertensive individuals with LDL-hypercholesterolemia. See text for further details. $L D L-C$ low-density lipoprotein cholesterol, PCSK9i PCSK9 inhibitors

or bempedoic acid plus ezetimibe as the initial lipid lowering strategy — might be conceivable also in the case of low to moderate cardiovascular risk, if the basal level of serum cholesterol is elevated, in order to avoid the use of the highest statin doses and improve adherence/persistence.

In this context, even limiting our attention to the high/ very high cardiovascular risk patients, the described approach suffers from some limitations and deserves some further comments. First, data on prevention of cardiovascular events with bempedoic acid are not yet available [56], while the efficacy of PCSK9is in reducing cardiovascular events has been tested in secondary prevention trials [11, $53,57,58]$. Despite their marked lipid-lowering efficacy and good safety profile, evidence in low- to medium-risk settings is minimal $[57,58]$. However, there are no reasons to believe that patients in primary prevention should derive different benefits from PCSK9 inhibition as compared to those in secondary prevention. In particular, the current evidence on the safety profile of PCSK9is in primary prevention is relatively scanty $[57,58]$. However, available data do not reveal any adverse effect $[57,58]$. Thus, the use of either 
alirocumab or evolocumab in hypertensive patients at high/ very high cardiovascular risk and no previous cardiovascular events is reasonably expected to be followed by a significant reduction in cardiovascular events and to maintain a good safety profile.

As a second concern, while the cost of the simultaneous prescription of a high intensity statin + ezetimibe - either as free or preferably as fixed combinations-is expected to be modestly higher, or not higher at all, than statin or ezetimibe monotherapies [59], the cost of bempedoic acid [60] in addition to ezetimibe and/or statins is expected to be higher than the cost of ezetimibe or statins alone. Nevertheless, among 1000 patients with high LDL cholesterol levels, bempedoic acid in addition to statin therapy is estimated to save 122 years of life and 103 quality-adjusted life years compared with statin therapy alone [60]. Thus, the additional cost of bempedoic acid \pm ezetimibe in the real world is very likely to be overwhelmed by the reduction in cardiovascular events and related costs. As a consequence, the use of high intensity statin+ezetimibe as a first step in the therapy in hypertensive hypercholesterolemic patients at high/very high ASCVD risk, with the possible addition of bempedoic acid and/or PCSK9is sounds reasonable and is likely to be followed by a more rapid and effective reduction in circulating LDL cholesterol levels than the use of statins alone.

Ongoing trials and novel drugs will allow further refinement of the proposed combined approach. A novel motto for such an approach would read like the following: "Our goal: achieve control. No setback: combine and check".

\section{Bullet Points}

- Focus on effective lifestyle modification for all patients.

- Fixed dose combination of high intensity statin plus ezetimibe as early first approach in patient with indication to pharmacological therapy.

- Introduction of bempedoic acid and/or PCSK9 inhibitors in patients not at target or intolerant to statins.

- Evinacumab and inclisiran are new drugs on the pipeline.

\section{Declarations}

Conflict of interests The authors declare no conflicts of interest.

Open Access This article is licensed under a Creative Commons Attribution-NonCommercial 4.0 International License, which permits any non-commercial use, sharing, adaptation, distribution and reproduction in any medium or format, as long as you give appropriate credit to the original author(s) and the source, provide a link to the Creative Commons licence, and indicate if changes were made. The images or other third party material in this article are included in the article's Creative Commons licence, unless indicated otherwise in a credit line to the material. If material is not included in the article's Creative Commons licence and your intended use is not permitted by statutory regulation or exceeds the permitted use, you will need to obtain permission directly from the copyright holder. To view a copy of this licence, visit http://creativecommons.org/licenses/by-nc/4.0/.

\section{References}

1. GBD 2019 Diseases and Injuries Collaborators. Global burden of 369 diseases and injuries in 204 countries and territories, 1990-2019: a systematic analysis for the Global Burden of Disease Study 2019. Lancet. 2020;396:1204-22.

2. Ford ES, Ajani UA, Croft JB, Critchley JA, Labarthe DR, Kottke TE, et al. Explaining the decrease in U.S. deaths from coronary disease, 1980-2000. N Engl J Med. 2007;356:2388-98.

3. Williams B, Masi S, Wolf J, Schmieder RE. Facing the challenge of lowering blood pressure and cholesterol in the same patient: report of a symposium at the European Society of Hypertension. Cardiol Ther. 2020;9:19-34.

4. Borghi C, Urso R, Cicero AF. Renin-angiotensin system at the crossroad of hypertension and hypercholesterolemia. Nutr Metab Cardiovasc Dis. 2017;27:115-20.

5. Koh KK, Sakuma I, Hayashi T, Kim SH, Chung W-J. Renin-angiotensin system inhibitor and statins combination therapeuticswhat have we learnt? Expert Opin Pharmacother. 2015;16:949-53.

6. Del Pinto R, Grassi D, Properzi G, Desideri G, Ferri C. Low Density Lipoprotein (LDL) cholesterol as a causal role for atherosclerotic disease: potential role of PCSK9 inhibitors. High Blood Press Cardiovasc Prev. 2019;26:199-207.

7. Brandts J, Ray KK. Bempedoic acid, an inhibitor of ATP citrate lyase for the treatment of hypercholesterolemia: early indications and potential. Expert Opin Investig Drugs. 2020;29:763-70.

8. Fitzgerald K, White S, Borodovsky A, Bettencourt BR, Strahs A, Clausen V, et al. A highly durable RNAi therapeutic inhibitor of PCSK9. N Engl J Med. 2017;376:41-51.

9. Evinacumab MA. First approval. Drugs. 2021;81:1101-5.

10. Grundy SM, Stone NJ, Bailey AL, Beam C, Birtcher KK, Blumenthal RS, et al. 2018 AHA/ACC/AACVPR/AAPA/ABC/ ACPM/ADA/AGS/APhA/ASPC/NLA/PCNA guideline on the management of blood cholesterol: executive summary: a report of the American College of Cardiology/American Heart Association Task Force on Clinical Practice Guidelines. Circulation. 2019;139:e1046-81.

11. Mach F, Baigent C, Catapano AL, Koskinas KC, Casula M, Badimon L, et al. 2019 ESC/EAS guidelines for the management of dyslipidaemias: lipid modification to reduce cardiovascular risk. Eur Heart J. 2020;41:111-88.

12. Miettinen TA. Diurnal variation of cholesterol precursors squalene and methyl sterols in human plasma lipoproteins. J Lipid Res. 1982;23:466-73.

13. Wallace A, Chinn D, Rubin G. Taking simvastatin in the morning compared with in the evening: randomised controlled trial. BMJ. 2003;327:788.

14. Cilla DD Jr, Gibson DM, Whitfield LR, Sedman AJ. Pharmacodynamic effects and pharmacokinetics of atorvastatin after administration to normocholesterolemic subjects in the morning and evening. J Clin Pharmacol. 1996;36:604-9.

15. Chasman DI, Giulianini F, MacFadyen J, Barratt BJ, Nyberg F, Ridker PM. Genetic determinants of statin-induced low-density lipoprotein cholesterol reduction: the Justification for the Use of Statins in Prevention: an Intervention Trial Evaluating Rosuvastatin (JUPITER) trial. Circ Cardiovasc Genet. 2012;5:257-64. 
16. Ward NC, Watts GF, Eckel RH. Statin toxicity. Circ Res. 2019;124:328-50.

17. Zhou Z, Curtis AJ, Breslin M, Nelson M. Letter by Zhou et al Regarding Article, "Statin Toxicity: Mechanistic Insights and Clinical Implications." Circ Res. 2019;124:e120.

18. Stroes ES, Thompson PD, Corsini A, Vladutiu GD, Raal FJ, Ray KK, et al. Statin-associated muscle symptoms: impact on statin therapy-European Atherosclerosis Society Consensus Panel Statement on Assessment, Aetiology and Management. Eur Heart J. 2015;36:1012-22.

19. SEARCH Collaborative Group, Link E, Parish S, Armitage J, Bowman L, Heath S, et al. SLCO1B1 variants and statin-induced myopathy - a genomewide study. N Engl J Med. 2008;359:789-99.

20. Siddiqui KM, Maroteau C, Veluchamy A, Tornio A, Tavendale R, Carr F, et al. A common missense variant of LILRB5 is associated with statin intolerance and myalgia. Eur Heart $\mathrm{J}$. 2017;38:3569-75.

21. Banach M, Stulc T, Dent R, Toth PP. Statin non-adherence and residual cardiovascular risk: there is need for substantial improvement. Int J Cardiol. 2016;225:184-96.

22. Website. https://www.aifa.gov.it/documents/20142/0/Rapporto_ OsMed_2018.pdf/. Accessed 04 Nov 2021.

23. Rosenson RS, Baker SK, Jacobson TA, Kopecky SL, Parker BA, The National Lipid Association's Muscle Safety Expert Panel. An assessment by the Statin Muscle Safety Task Force: 2014 update. J Clin Lipidol. 2014;8:S58-71.

24. Sudhop T, Lütjohann D, Kodal A, Igel M, Tribble DL, Shah S, et al. Inhibition of intestinal cholesterol absorption by ezetimibe in humans. Circulation. 2002;106:1943-8.

25. Morrone D, Weintraub WS, Toth PP, Hanson ME, Lowe RS, Lin $\mathrm{J}$, et al. Lipid-altering efficacy of ezetimibe plus statin and statin monotherapy and identification of factors associated with treatment response: a pooled analysis of over 21,000 subjects from 27 clinical trials. Atherosclerosis. 2012;223:251-61.

26. Jones MR, Nwose OM. Role of colesevelam in combination lipidlowering therapy. Am J Cardiovasc Drugs. 2013;13:315-23.

27. Nissen SE, Stroes E, Dent-Acosta RE, Rosenson RS, Lehman SJ, Sattar N, et al. Efficacy and tolerability of evolocumab vs ezetimibe in patients with muscle-related statin intolerance: the GAUSS-3 randomized clinical trial. JAMA. 2016;315:1580-90.

28. Cannon CP, Blazing MA, Giugliano RP, McCagg A, White JA, Theroux $P$, et al. Ezetimibe added to statin therapy after acute coronary syndromes. N Engl J Med. 2015;372:2387-97.

29. Myocardial Infarction Genetics Consortium Investigators, Stitziel NO, Won H-H, Morrison AC, Peloso GM, Do R, et al. Inactivating mutations in NPC1L1 and protection from coronary heart disease. N Engl J Med. 2014;371:2072-82.

30. Rosenson RS, Hegele RA, Fazio S, Cannon CP. The evolving future of PCSK9 inhibitors. J Am Coll Cardiol. 2018;72:314-29.

31. Brown MS, Anderson RG, Goldstein JL. Recycling receptors: the round-trip itinerary of migrant membrane proteins. Cell. 1983;32:663-7.

32. Thedrez A, Blom DJ, Ramin-Mangata S, Blanchard V, Croyal M, Chemello K, et al. Homozygous familial hypercholesterolemia patients with identical mutations variably express the LDLR (low-density lipoprotein receptor): implications for the efficacy of evolocumab. Arterioscler Thromb Vasc Biol. 2018;38:592-8.

33. Sabatine MS, Giugliano RP, Keech AC, Honarpour N, Wiviott SD, Murphy SA, et al. Evolocumab and clinical outcomes in patients with cardiovascular disease. N Engl J Med. 2017;376:1713-22.

34. Talasaz AH, Ho A-C, Bhatty F, Koenig RA, Dixon DL, Baker WL, et al. Meta-analysis of clinical outcomes of PCSK9 modulators in patients with established ASCVD. Pharmacotherapy. 2021. https://doi.org/10.1002/phar.2635.
35. Nair JK, Willoughby JLS, Chan A, Charisse K, Alam MR, Wang Q, et al. Multivalent $N$-acetylgalactosamine-conjugated siRNA localizes in hepatocytes and elicits robust RNAi-mediated gene silencing. J Am Chem Soc. 2014;136:16958-61.

36. Ray KK, Landmesser U, Leiter LA, Kallend D, Dufour R, Karakas $\mathrm{M}$, et al. Inclisiran in patients at high cardiovascular risk with elevated LDL cholesterol. N Engl J Med. 2017;376:1430-40.

37. Ray KK, Wright RS, Kallend D, Koenig W, Leiter LA, Raal FJ, et al. Two phase 3 trials of inclisiran in patients with elevated LDL cholesterol. N Engl J Med. 2020;382:1507-19.

38. A randomized trial assessing the effects of inclisiran on clinical outcomes among people with cardiovascular disease-full text view-ClinicalTrials.gov. https://clinicaltrials.gov/ct2/show/ NCT03705234. Accessed 21 Oct 2021.

39. Raal FJ, Rosenson RS, Reeskamp LF, Hovingh GK, Kastelein JJP, Rubba P, et al. Evinacumab for homozygous familial hypercholesterolemia. N Engl J Med. 2020;383:711-20.

40. Rosenson RS, Burgess LJ, Ebenbichler CF, Baum SJ, Stroes ESG, Ali S, et al. Evinacumab in patients with refractory hypercholesterolemia. N Engl J Med. 2020;383:2307-19.

41. Fujimoto K, Koishi R, Shimizugawa T, Ando Y. Angptl3-null mice show low plasma lipid concentrations by enhanced lipoprotein lipase activity. Exp Anim. 2006;55:27-34.

42. Dewey FE, Gusarova V, Dunbar RL, O’Dushlaine C, Schurmann $\mathrm{C}$, Gottesman $\mathrm{O}$, et al. Genetic and pharmacologic inactivation of ANGPTL3 and cardiovascular disease. N Engl J Med. 2017;377:211-21.

43. Banerjee P, Chan K-C, Tarabocchia M, Benito-Vicente A, Alves AC, Uribe KB, et al. Functional analysis of LDLR (low-density lipoprotein receptor) variants in patient lymphocytes to assess the effect of evinacumab in homozygous familial hypercholesterolemia patients with a spectrum of LDLR activity. Arterioscler Thromb Vasc Biol. 2019;39:2248-60.

44. Gaudet D, Gipe DA, Pordy R, Ahmad Z, Cuchel M, Shah PK, et al. ANGPTL3 inhibition in homozygous familial hypercholesterolemia. N Engl J Med. 2017;377:296-7.

45. Bays HE, Banach M, Catapano AL, Duell PB, Gotto AM Jr, Laufs $\mathrm{U}$, et al. Bempedoic acid safety analysis: pooled data from four phase 3 clinical trials. J Clin Lipidol. 2020;14:649-659.e6.

46. Evaluation of major cardiovascular events in patients with, or at high risk for, cardiovascular disease who are statin intolerant treated with bempedoic acid (ETC-1002) or placebo-full text view-ClinicalTrials.gov. https://clinicaltrials.gov/ct2/show/ NCT02993406. Accessed 21 Oct 2021.

47. Ballantyne CM, Banach M, Mancini GBJ, Lepor NE, Hanselman JC, Zhao X, et al. Efficacy and safety of bempedoic acid added to ezetimibe in statin-intolerant patients with hypercholesterolemia: a randomized, placebo-controlled study. Atherosclerosis. 2018;277:195-203.

48. Laufs U, Banach M, Mancini GBJ, Gaudet D, Bloedon LT, Sterling LR, et al. Efficacy and safety of bempedoic acid in patients with hypercholesterolemia and statin intolerance. J Am Heart Assoc. 2019;8: e011662.

49. Goldberg AC, Leiter LA, Stroes ESG, Baum SJ, Hanselman JC, Bloedon LT, et al. Effect of bempedoic acid vs placebo added to maximally tolerated statins on low-density lipoprotein cholesterol in patients at high risk for cardiovascular disease: the CLEAR wisdom randomized clinical trial. JAMA. 2019;322:1780-8.

50. Ray KK, Bays HE, Catapano AL, Lalwani ND, Bloedon LT, Sterling LR, et al. Safety and efficacy of bempedoic acid to reduce LDL cholesterol. N Engl J Med. 2019;380:1022-32.

51. Cicero AFG, Grassi D, Tocci G, Galletti F, Borghi C, Ferri C. Nutrients and nutraceuticals for the management of high normal blood pressure: an evidence-based consensus document. High Blood Press Cardiovasc Prev. 2019;26:9-25. 
52. Volpe M, Volpe R, Gallo G, Presta V, Tocci G, Folco E, et al. 2017 Position paper of the Italian Society for Cardiovascular Prevention (SIPREC) for an updated clinical management of hypercholesterolemia and cardiovascular risk: executive document. High Blood Press Cardiovasc Prev. 2017;24:313-29.

53. Visseren FLJ, Mach F, Smulders YM, Carballo D, Koskinas KC, Bäck M, et al. 2021 ESC guidelines on cardiovascular disease prevention in clinical practice. Eur Heart J. 2021;42:3227-337.

54. Williams B, Mancia G, Spiering W, Agabiti Rosei E, Azizi M, Burnier M, et al. 2018 ESC/ESH guidelines for the management of arterial hypertension. Eur Heart J. 2018;39:3021-104.

55. Maloberti A, Giannattasio C, Bombelli M, Desideri G, Cicero AFG, Muiesan ML, et al. Hyperuricemia and risk of cardiovascular outcomes: the experience of the URRAH (Uric Acid Right for Heart Health) project. High Blood Press Cardiovasc Prev. 2020;27:121-8.

56. Website. Available: Evaluation of major cardiovascular events in patients with, or at high risk for, cardiovascular disease who are statin intolerant treated with bempedoic acid (ETC-1002) or placebo-full text view-ClinicalTrials.gov. https://clinicaltrials. gov/ct2/show/NCT02993406. Accessed 21 Oct 2021.

57. Pokhrel B, Yuet WC, Levine SN. PCSK9 inhibitors. Treasure Island: StatPearls Publishing; 2021.

58. Schmidt AF, Carter J-PL, Pearce LS, Wilkins JT, Overington JP, Hingorani AD, et al. PCSK9 monoclonal antibodies for the primary and secondary prevention of cardiovascular disease. Cochrane Database Syst Rev. 2020;10: CD011748.

59. Fras Z, Mikhailidis DP. Have we learnt all from IMPROVE-IT? Part II. Subanalyses of the effects of ezetimibe added to statin therapy on selected clinical and laboratory outcomes, cost-effectiveness, guidelines, and clinical implications. Curr Vasc Pharmacol. 2021;19:469-86.

60. Perera K, Kam N, Ademi Z, Liew D, Zomer E. Bempedoic acid for high-risk patients with CVD as adjunct lipid-lowering therapy: a cost-effectiveness analysis. J Clin Lipidol. 2020;14:772-83. 